\title{
Cross-Document Pattern Matching
}

\author{
Gregory Kucherov $^{1}$, Yakov Nekrich ${ }^{2}$, and Tatiana Starikovskaya ${ }^{3,1}$ \\ 1 Laboratoire d'Informatique Gaspard Monge, Université Paris-Est \& CNRS, Marne-la-Vallée, Paris, \\ France, Gregory.Kucherov@univ-mlv.fr \\ 2 Department of Computer Science, University of Chile, yakov.nekrich@googlemail.com \\ 3 Lomonosov Moscow State University, Moscow, Russia, tat.starikovskaya@gmail.com
}

\begin{abstract}
We study a new variant of the string matching problem called cross-document string matching, which is the problem of indexing a collection of documents to support an efficient search for a pattern in a selected document, where the pattern itself is a substring of another document. Several variants of this problem are considered, and efficient linear-space solutions are proposed with query time bounds that either do not depend at all on the pattern size or depend on it in a very limited way (doubly logarithmic). As a side result, we propose an improved solution to the weighted level ancestor problem.
\end{abstract}

\section{Introduction}

In this paper we study the following variant of the string matching problem that we call cross-document string matching: given a collection of strings (documents) stored in a "database", we want to be able to efficiently search for a pattern in a given document, where the pattern itself is a substring of another document. More formally, assuming we have a set of documents $T_{1}, \ldots, T_{m}$, we want to answer queries about the occurrences of a substring $T_{k}[i . . j]$ in a document $T_{\ell}$.

This scenario may occur in various practical situations when we have to search for a pattern in a text stored in a database, and the pattern is itself drawn from a string from the same database. This is a common situation in bioinformatics, where one may want to repeatedly look for genomic elements drawn from a genome within a set of genomic sequences involved in the project. In bibliographic search, it is common to look up words or citations coming from one document in other documents. Similar scenarios may occur in other traditional applications of string matching, such as in the analysis of web server logfiles for example.

In this paper, we study different versions of the cross-document string matching problem. First, we distinguish between counting and reporting queries, asking respectively about the number of occurrences of $T_{k}[i . . j]$ in $T_{\ell}$ or about the occurrences themselves. The two query types lead to slightly different solutions. In particular, the counting problem uses the weighted level ancestor problem $[10,1]$ to which we propose a new solution with an improved complexity bound.

We further consider different variants of the two problems. The first one is the dynamic version where new documents can be added to the database. In another variant, called document counting and reporting, we only need to respectively count or report the documents 
containing the pattern, rather than counting or reporting pattern occurrences within a given document. This version is very close to the document retrieval problem previously studied (see [15] and later papers referring to it), with the difference that in our case the pattern is itself selected from the documents stored in the database. Finally, we also consider succinct data structures for the above problems, where we keep involved index data structure in compressed form.

Let $m$ be the number of stored strings and $n$ the total length of all strings. Our results are summarized below:

(i) for the counting problem, we propose a solution with query time $O(t+\log \log m)$, where $t=\min (\sqrt{\log \text { occ } / \log \log \text { occ }}, \log \log |P|), P=T_{k}[i . . j]$ is the searched substring and occ is the number of its occurrences in $T_{\ell}$,

(ii) for the reporting problem, our solution outputs all the occurrences in time $O(\log \log m+$ occ),

(iii) in the dynamic case, when new documents can be dynamically added to the database, we are able to answer counting queries in time $O(\log n)$ and reporting queries in time $O(\log n+$ occ $)$, whereas the updates take time $O(\log n)$ per character,

(iv) for the document counting and document reporting problems, our algorithms run in time $O(\log n)$ and $O(t+$ ndocs $)$ respectively, where $t$ is as above and ndocs is the number of reported documents,

(v) finally, we also present succinct data structures that support counting, reporting, and document reporting queries in cross-document scenario (see Theorems 6 and 7 in Section 4.3).

For problems (i)-(iv), the involved data structures occupy $O(n)$ space. Interestingly, in the cross-document scenario, the query times either do not depend at all on the pattern length or depend on it in a very limited (doubly logarithmic) way.

Throughout the paper positions in strings are numbered from 1 . Notation $T[i . . j]$ stands for the subword $T[i] T[i+1] \ldots T[j]$ of $T$, and $T[i .$.$] denotes the suffix of T$ starting at position $i$.

\section{Preliminaries}

\subsection{Basic Data Stuctures}

We assume a basic knowledge of suffix trees and suffix arrays.

Besides using suffix trees for individual strings $T_{i}$, we will also be using the generalized suffix tree for a set of strings $T_{1}, T_{2}, \ldots, T_{m}$ that can be viewed as the suffix tree for the string $T_{1} \$_{1} T_{2} \$_{2} \ldots T_{m} \$_{m}$. A leaf in a suffix tree for $T_{i}$ is associated with a distinct suffix of $T_{i}$, and a leaf in the generalized suffix tree is associated with a suffix of some document $T_{i}$ together with the index $i$ of this document. We assume that for each node $v$ of a suffix tree, the number $n_{v}$ of leaves in the subtree rooted at $v$, as well as its string depth $d(v)$ can be recovered in constant time. Recall that the string depth $d(v)$ is the total length of strings labelling the edges along the path from the root to $v$. 
We will also use the suffix arrays for individual documents as well as the generalized suffix array for strings $T_{1}, T_{2}, \ldots, T_{m}$. Each entry of the suffix array for $T_{i}$ is associated with a distinct suffix of $T_{i}$ and each entry of the generalized suffix array for $T_{1}, \ldots, T_{m}$ is associated with a suffix of some document $T_{i}$ and the index $i$ of the document the suffix comes from. We store these document indices in a separate array $D$, called document array, such that $D[i]=k$ if the $i$-th entry of the generalized suffix array for $T_{1}, \ldots, T_{m}$ corresponds to a suffix coming from $T_{k}$.

For each considered suffix array, we assume available, when needed, two auxiliary arrays: an inverted suffix array and another array, called the LCP-array, of longest common prefixes between each suffix and the preceding one in the lexicographic order.

Suffix trees and suffix arrays are naturally related: if the children of any internal node of a suffix tree are ordered in the lexicographic order of the labels (actually, of the first symbols of the labels, as they are all distinct), then the leaves ordered "left-to-right" correspond exactly to the suffix array with respect to the referred suffixes.

\subsection{Weighted Level Ancestor Problem}

The weighted level ancestor problem, defined in [10], is a generalization of the level ancestor problem $[6,5]$ for the case when tree edges are assigned positive weights.

Consider a rooted tree $\mathcal{T}$ whose edges are assigned positive integer weights. For a node $w$, let weight $(w)$ denote the total weight of the edges on the path from the root to $w$. $\operatorname{depth}(w)$ denotes the usual tree depth of $w$. A weighted level ancestor query wla $(v, q)$ asks, given a node $v$ and a positive integer $q$, for the ancestor $w$ of $v$ of minimal depth such that weight $(w) \geq q($ wla $(v, q)$ is undefined if there is no such node $w)$.

Two previously known solutions $[10,1]$ for weighted level ancestors problem achieve $O(\log \log W)$ query time using linear space, where $W$ is the total weight of all tree edges. Our data structure also uses $O(n)$ space, but achieves a faster query time in many special cases. We prove the following result.

Theorem 1. There exists an $O(n)$ space data structure that answers weighted ancestor query wla $(v, q)$ in $O(\min (\sqrt{\log g / \log \log g}, \log \log q))$ time, where $g=$ $\min (\operatorname{depth}(\operatorname{wla}(v, q)), \operatorname{depth}(v)-\operatorname{depth}(w \mathrm{wla}(v, q)))$.

If every internal node is a branching node, we obtain the following corollary.

Corollary 1. Suppose that every internal node in $\mathcal{T}$ has at least two children. There exists an $O(n)$ space data structure that finds $w=\operatorname{wla}(v, q)$ in $O\left(\sqrt{\log n_{w} / \log \log n_{w}}\right)$ time, where $n_{w}$ is the number of leaves in the subtree of $w$.

Our approach combines the heavy path decomposition technique of [1] with efficient data structures for finger searching in a set of integers. Due to space limitations, the proof is given in the Appendix. 


\section{Cross-document Pattern Counting and Reporting}

\subsection{Counting}

In this section we consider the problem of counting occurrences of a pattern $T_{k}[i . . j]$ in a document $T_{\ell}$.

Our data structure consists of the generalized suffix array $G S A$ for documents $T_{1}, \ldots, T_{m}$ and individual suffix trees $\mathcal{T}_{i}$ for every document $T_{i}$. We assume that entries of $G S A$ and leaves of suffix trees $\mathcal{T}_{i}$ are linked by pointers so that given the location of some suffix $T_{k}[i .$. in $G S A$, we can retrieve its position in $\mathcal{T}_{k}$.

For every suffix tree $\mathcal{T}_{\ell}$ we store a data structure of Theorem 1 supporting weighted level ancestor queries on $\mathcal{T}_{\ell}$. We also augment the document array $D$ with a $O(n)$-space data structure that answers queries $\operatorname{rank}(k, i)$ (number of entries storing $k$ before position $i$ in $D)$ and select $(k, i)$ ( $i$-th entry from the left storing $k$ ). Using the result of [13], we can support such rank and select queries in $O(\log \log m)$ and $O(1)$ time respectively. Moreover, we construct a data structure that answers range minima queries (RMQ) on the $L C P$ array: for any $1 \leq r_{1} \leq r_{2} \leq n$, find the minimum among $L C P\left[r_{1}\right], \ldots L C P\left[r_{2}\right]$. There exists a linear space RMQ data structure that supports queries in constant time, see e.g., [4]. An RMQ query on the $L C P$ array computes the length of the longest common prefix of two suffixes $G S A\left[r_{1}\right]$ and $G S A\left[r_{2}\right]$, denoted $L C P\left(r_{1}, r_{2}\right)$.

Our counting algorithm consists of two stages. First, using $G S A$, we identify a position $p$ of $T_{\ell}$ at which the query pattern $T_{k}[i . . j]$ occurs, or determine that no such $p$ exists. Then we find the locus of $T_{k}[i . . j]$ in the suffix tree $\mathcal{T}_{\ell}$ using a weighted ancestor query.

Let $r$ be the position of $T_{k}[i .$.$] in the G S A$. We find indexes $r_{1}=\operatorname{select}(\ell, \operatorname{rank}(r, \ell))$ and $r_{2}=\operatorname{select}(\ell, \operatorname{rank}(r, \ell)+1)$ in $O(\log \log m)$ time. $G S A\left[r_{1}\right]\left(\operatorname{resp} . G S A\left[r_{2}\right]\right)$ is the closest suffix from document $T_{\ell}$ that precedes (resp. follows) $T_{k}[i .$.$] in the lexicographic order of$ suffixes. Observe now that $T_{k}[i . . j]$ occurs in $T_{\ell}$ if and only if either $\operatorname{LCP}\left(r_{1}, r\right)$ or $\operatorname{LCP}\left(r, r_{2}\right)$ (or both) is no less than $j-i+1$. If this holds, then the starting position $p$ of $G S A\left[r_{1}\right]$ (respectively, starting position of $G S A\left[r_{2}\right]$ ) is the position of $T_{k}[i . . j]$ in $T_{\ell}$. Once such a position $p$ is found, we jump to the corresponding leaf $T_{\ell}[p .$.$] in the suffix tree of T_{\ell}$.

Let $v$ be the leaf of $\mathcal{T}_{\ell}$ that contains the suffix $T_{\ell}[p .$.$] . Then the weighted level ancestor$ $u=\operatorname{wla}(v,(j-i+1))$ is the locus of $T_{k}[i . . j]$ in $\mathcal{T}_{\ell}$. This is because $T_{\ell}[p . . p+j-i]=T_{k}[i . . j]$. By Corollary 1, we can find node $u$ in $O\left(\sqrt{\log n_{u} / \log \log n_{u}}\right)$ time, where $n_{u}$ is the number of leaf descendants of $u$. Since $u$ is the locus node of $T_{k}[i . . j], n_{u}$ is the number of occurrences of $T_{k}[i . . j]$ in $T_{\ell}$. By Theorem 1, we can find $u$ in $O(\log \log (j-i+1))$ time.

Summing up, we obtain the following Theorem.

Theorem 2. For any $1 \leq k, \ell \leq m$ and $1 \leq i \leq j \leq\left|T_{k}\right|$, we can count the number of occurrences of $T_{k}[i . . j]$ in $T_{\ell}$ in $O(t+\log \log m)$ time, where $t=$ $\min (\sqrt{\log \text { occ } / \log \log \text { occ }}, \log \log (j-i+1))$ and occ is the number of occurrences. The underlying indexing structure takes $O(n)$ space and can be constructed in $O(n)$ time.

Observe that our data structure always answers range counting queries in $O(\log \log n)$ time. If $m$ and either the pattern length $(j-i+1)$ or the number of occurrences are sufficiently 
small, the query time is even better. For instance if $m=O(1)$ and occ $=O(1)$, a query is answered in constant time.

\subsection{Reporting}

A reporting query asks for all occurrences of a substring $T_{k}[i . . j]$ in $T_{\ell}$.

Compared to counting queries, we make a slight change in the data structures: instead of using suffix trees for individual documents $T_{i}$, we use suffix arrays. Similarly to the previous section, we link each entry of $G S A$ to a corresponding entry in the corresponding individual suffix array. The rest of the data structures is unchanged.

We first find an occurrence of $T_{k}[i . . j]$ in $T_{\ell}$ (if there is one) with the method described in Section 3.1. Let $p$ be the position of this occurrence in $T_{\ell}$. We then jump to the corresponding entry $r$ of the suffix array $S A_{\ell}$ for the document $T_{\ell}$. Let $L C P_{\ell}$ be the LCP-array of $S A_{\ell}$. Starting with entry $r$, we visit adjacent entries $t$ of $S A_{\ell}$ moving both to the left and to the right as long as $L C P_{\ell}[t] \geq j-i+1$. While this holds, we report $S A_{\ell}[t]$ as an occurrence of $T_{k}[i . . j]$. It is easy to observe that the procedure is correct and that no occurrence is missing. As a result, we obtain the following theorem.

Theorem 3. All the occurrences of $T_{k}[i . . j]$ in $T_{\ell}$ can be reported in $O(\log \log m+$ occ $)$ time, where occ is the number of occurrences. The underlying indexing structure takes $O(n)$ space and can be constructed in $O(n)$ time.

Observe that the algorithm has no dependence whatsoever on the pattern length, and that the query time does not depend on the length of documents but only on their number.

\section{Variants of the Problem}

\subsection{Dynamic Counting and Reporting}

In this section we focus on a dynamic version of counting and reporting problems, where the only dynamic operation consists in adding a document to the database ${ }^{4}$.

Recall that in the static case, counting occurrences of $T_{k}[i . . j]$ in $T_{\ell}$ is done through the following two steps (Section 3.1):

1. compute position $p$ of some occurrence of $T_{k}[i . . j]$ in $T_{\ell}$,

2. in the suffix tree of $T_{\ell}$, find the locus of string $T_{\ell}[p . . p+j-i]$, and retrieve the number of leaves in the subtree rooted at $u$.

For reporting queries (Section 3.2), Step 1 is basically the same, while Step 2 is different and uses an individual suffix array for $T_{\ell}$.

In the dynamic framework, we follow the same general two-step scenario. Note first that since Step 2, for both counting and reporting, uses data structures for individual

\footnotetext{
${ }^{4}$ document deletions are also possible to support but require some additional constructions that are left to the extended version of this paper
} 
documents only, it trivially applies to the dynamic case without changes. However, Step 1 requires serious modifications that we describe below.

Since the suffix array is not well-suited for dynamic updates, at Step 1 we will use the generalized suffix tree for $T_{1}, T_{2}, \ldots, T_{m}$ hereafter denoted $G S T$. For each suffix of $T_{1}, T_{2}, \ldots, T_{m}$ we store a pointer to the leaf of $G S T$ corresponding to this suffix. Unfortunately, it is not easy to maintain the lexicographically ordered list of suffixes when $G S T$ is dynamically updated, as it is not easy to quickly determine the location of a newly created leaf in the list. Another task to be solved is to support updates of LCP-values and range minima queries on these values ${ }^{5}$.

To this end, we introduce the following additional data structure. We maintain a dynamic doubly-linked list corresponding to the Euler tour of the current GST. This list is denoted by $E L$. Each internal node of $G S T$ is stored in two copies in $E L$, corresponding respectively to the first and last visits of the node during the Euler tour. Leaves of GST are kept in one copy. Observe that the leaves of GST appear in $E L$ in the same "left-to-right" order, although not consecutively.

On $E L$, we maintain the data structure of [3] which allows, given two list elements, to determine their order in the list in $O(1)$ time (see also [9]). Insertions of elements in the list are supported in $O(1)$ time too.

Furthermore, we maintain a balanced tree, denoted $B T$, whose leaves are elements of $E L$. Note that the size of $E L$ is bounded by $2 n$ ( $n$ is the size of GST) and the height of $B T$ is $O(\log n)$. Since the leaves of $G S T$ are a subset of the leaves of $B T$, we call them suffix leaves to avoid the ambiguity.

Each internal node $u$ of $B T$ stores two kinds of information: (i) the rightmost and leftmost suffix leaves in the subtree of $B T$ rooted at $u$, (ii) minimal LCP value among all suffix leaves in the subtree of $B T$ rooted at $u$.

Finally, we will also need an individual suffix array for each inserted document $T_{i}$.

We are now in position to describe the algorithm of Step 1. Like in the static case, we first retrieve the leaf of GST corresponding to suffix $T_{k}[i .$.$] . To identify a position of an$ occurrence of $T_{k}[i . . j]$ in $T_{\ell}$, we have to examine the two closest elements in the list of leaves of GST, one from right and from left, corresponding to suffixes of $T_{\ell}$. To find these two suffixes, we perform a binary search on the suffix array for $T_{\ell}$ using order queries of [3] on $E L$. This step takes $O\left(\log \left|T_{\ell}\right|\right)$ time.

We then check if at least one of these two suffixes corresponds to an occurrence of $T_{k}[i . . j]$ in $T_{\ell}$. In a similar way to Section 3 , we have to compute the longest common prefix between each of these two suffixes and $T_{k}[i .$.$] , and compare this value with (j-i+1)$. This amounts to computing the minimal $L C P$ value among all the suffixes of the corresponding range, i.e. to answering a range-minima query. To do this, we resort to the list $E L$ and the tree $B T$ and use the standard technique used for answering range queries: for any sublist $L^{\prime}$ of $E L$ we can identify $O(\log n)$ nodes $v_{i}$ of $B T$, so that an element $e$ belongs to $L^{\prime}$ if and only if it is a leaf descendant of some node $v_{i}$. We retrieve $O(\log n)$ nodes $v_{i}$ that cover the

\footnotetext{
${ }^{5}$ supporting dynamic RMQ could be done with the general method of [8], however we will give here a simpler ad hoc algorithm with the same time complexity, which is sufficient for our purposes
} 
relevant sublist of $E L$. The least among all minimal $L C P$ values stored in nodes $v_{i}$ is the minimal LCP value for the specified range of suffixes. Thus, computing the length of the longest common prefix of two suffixes takes $O(\log n)$ time. Once a witness occurrence of $T_{k}[i . . j]$ in $T_{\ell}$ is found, Step 2 is done as explained in Sections 3.1,3.2.

The query time bounds are summarized in the following lemma.

Lemma 1. Using the above data structures, counting and reporting all occurrences of $T_{k}[i . . j]$ in $T_{\ell}$ can be done respectively in time $O(\log n)$ and time $O(\log n+$ occ $)$, where occ is the number of reported occurrences.

We now explain how the involved data structures are updated. Suppose that we add a new document $T_{m+1}$. Extending the generalized suffix tree by $T_{m+1}$ is done in time $O\left(\left|T_{m+1}\right|\right)$ by McCreight's or Ukkonen's algorithm, i.e. in $O(1)$ amortized time per symbol.

When a new node $v$ is added to a suffix tree, the following updates should be done (in order):

(i) insert $v$ at the right place of the list $E L$ (in two copies if $v$ is an internal node),

(ii) rebalance the tree $B T$ if needed,

(iii) if $v$ is a leaf of $G S T$ (i.e. a suffix leaf of $B T$ ), update LCP values and rightmost/leftmost suffix leaf information in $B T$,

To see how update (i) works, we have to recall how suffix tree is updated when a new document is inserted. Two possible updates are creation of a new internal node $v$ by splitting an edge into two (edge subdivision) and creating a new leaf $u$ as a child of an existing node. In the first case, we insert the first copy of $v$ right after the first copy of its parent, and the second copy right before the second copy of its parent. In the second case, the parent of $u$ has already at least one child, and we insert $u$ either right after the second (or the only) copy of its left sibling, or right before the first (or the only) copy of its right sibling.

Rebalancing the tree $B T$ (update (ii)) is done using standard methods. Observe that during the rebalancing we may have to adjust the LCP and rightmost/leftmost suffix leaf information for internal nodes, but this is easy to do as only a finite number of local modifications is done at each level.

Update (iii) is triggered when a new leaf $u$ is created in GST and added to EL. First of all, we have to compute the $L C P$ value for $u$ and possibly to update the $L C P$ value of the next suffix leaf $u^{\prime}$ to the right of $u$ in $E L$. This is done in $O(1)$ time as follows. At the moment when $u$ is created, we memorize the string depth of its parent $D=d(\operatorname{parent}(u))$. Recall that the parent of $u$ already has at least one child before $u$ is created. If $u$ is neither the leftmost nor the rightmost child of its parent, then we set $L C P(u)=D$ and $L C P\left(u^{\prime}\right)$ remains unchanged (actually it also equals $D$ ). If $u$ is the leftmost child of its parent, then we set $L C P(u)=L C P\left(u^{\prime}\right)$ and then $L C P\left(u^{\prime}\right)=D$. Finally, if $u$ is the rightmost child, then $L C P(u)=D$ and $L C P\left(u^{\prime}\right)$ remains unchanged.

We then have to follow the path in $B T$ from the new leaf $u$ to the root and possibly update the LCP and rightmost/leftmost suffix leaf information for all nodes on this path. These updates are straightforward. Furthermore, during this traversal we also identify suffix 
leaf $u^{\prime}$ (as the leftmost child of the first right sibling encountered during the traversal), update its $L C P$ value and, if necessary, the $L C P$ values on the path from $u^{\prime}$ to the root of $B T$. All these steps take time $O(\log n)$.

Thus, updates of all involved data structures take $O(\log n)$ time per symbol. The following theorem summarizes the results of this section.

Theorem 4. In the case when documents can be added dynamically, the number of occurrences of $T_{k}[i . . j]$ in $T_{\ell}$ can be computed in time $O(\log n)$ and reporting these occurrences can be done in time $O(\log n+$ occ $)$, where occ is their number. The underlying data structure occupies $O(n)$ space and an update takes $O(\log n)$ time per character.

\subsection{Document Counting and Reporting}

Consider a static collection of documents $T_{1}, \ldots, T_{m}$. In this section we focus on document reporting and counting queries: report or count the documents which contain at least one occurrence of $T_{k}[i . . j]$, for some $1 \leq k \leq m$ and $i \leq j$.

For both counting and reporting, we use the generalized suffix tree, generalized suffix array and the document array $D$ for $T_{1}, T_{2}, \ldots, T_{m}$. We first retrieve the leaf of the generalized suffix tree labelled by $T_{k}[i .$.$] and compute its highest ancestor u$ of string depth at least $j-i+1$, using the weighted level ancestor technique of Section 2.2. The suffixes of $T_{1}, T_{2}, \ldots, T_{m}$ starting with $T_{k}[i . . j]$ (i.e. occurrences of $T_{k}[i . . j]$ ) correspond then to the leaves of the subtree rooted at $u$, and vice versa. As shown in section 3.1, this step takes $O(t)$ time, where $t=\min (\sqrt{\log \text { occ } / \log \log \text { occ }}, \log \log (j-i+1))$ and occ is the number of occurrences of $T_{k}[i . . j]$ (this time in all documents).

Once $u$ has been computed, we retrieve the interval [left(u)..right $(u)]$ of ranks of all the leaves under interest. We are then left with the problem of counting/reporting distinct values in $D[$ left $(u) . . r i g h t(u)]$. This problem is exactly the same as the color counting/ color reporting problem that has been studied extensively (see e.g., [12] and references therein).

For color reporting queries, we can use the solution of [15] based on a $O(n)$-space data structure for RMQ, applied to (a transform of) the document array $D$. The pre-processing time is $O(n)$. Each document is then reported in $O(1)$ time, i.e. all relevant documents are reported in $O$ (ndocs) time, where ndocs is their number. The whole reporting query then takes time $O(t+$ ndocs $)$ for $t$ defined above.

For counting, we use the solution described in [7]. The data structure requires $O(n)$ space and a color counting query takes $O(\log n)$ time. The following theorem presents a summary.

Theorem 5. We can store a collection of documents $T_{1}, \ldots, T_{m}$ in a linear space data structure, so that for any pattern $P=T_{k}[i . . j]$ all documents that contain $P$ can be reported and counted in $O(t+$ ndocs $)$ and $O(\log n)$ time respectively. Here $t=$ $\min (\sqrt{\log \text { occ } / \log \log \text { occ }}, \log \log |P|)$, ndocs is the number of documents that contain $P$ and occ is the number of occurrences of $P$ in all documents.

Again, our query time does not depend on the pattern length, or this dependency is reduced. 


\subsection{Compact Counting, Reporting and Document Reporting}

In this section, we show how our reporting and counting probems can be solved on succinct data structures [16].

Reporting and Counting. Our compact solution is based on compressed suffix arrays [14]. A compressed suffix array for a text $T$ uses $|C S A|$ bits of space and enables us to retrieve the position of the suffix of rank $r$, the rank of a suffix $T[i .$.$] , and the char-$ acter $T[i]$ in time $\operatorname{Lookup}(n)$. Different trade-offs between space usage and query time can be achieved (see [16] for a survey).

Our data structure consists of a compressed generalized suffix array $C S A$ for $T_{1}, \ldots, T_{m}$ and compressed suffix arrays $C S A_{i}$ for each document $T_{i}$. In [17] it was shown that using $O(n)$ extra bits, the length of the longest common prefix of any two suffixes can be computed in $O(\operatorname{Lookup}(n))$ time. Besides, the ranks of any two suffixes $T_{k}[s .$.$] and T_{\ell}[p .$.$] can be$ compared in $O(\operatorname{Lookup}(n))$ time: it suffices to compare $T_{\ell}[p+f]$ with $T_{k}[s+f]$ for $f=$ $\operatorname{LCP}\left(T_{k}[s .],. T_{\ell}[p .].\right)$.

Note that ranks of the suffixes of $T_{\ell}$ starting with $T_{k}[i . . j]$ form an interval $\left[r_{1}, r_{2}\right]$. We use a binary search on the compressed suffix array of $T_{\ell}$ to find $r_{1}$ and $r_{2}$. At each step of the binary search we compare a suffix of $T_{\ell}$ with $T_{k}[i .$.$] . Therefore \left[r_{1}, r_{2}\right]$ can be found in $O(\operatorname{Lookup}(n) \cdot \log n)$ time. Obviously, the number of occurrences of $T_{k}[i . . j]$ in $T_{\ell}$ is $r_{2}-r_{1}$. To report the occurrences, we compute the suffixes of $T_{\ell}$ with ranks in interval $\left[r_{1}, r_{2}\right]$.

Theorem 6. All occurrences of $T_{k}[i . . j]$ in $T_{\ell}$ can be counted in $O(\operatorname{Lookup}(n) \cdot \log n)$ time and reported in $O((\log n+\mathrm{occ})$ Lookup $(n))$ time, where occ is the number of those. The underlying indexing structure takes $2|C S A|+O\left(n+m \log \frac{n}{m}\right)$ bits of memory.

Document Reporting Again, we use a binary search on the generalized suffix array to find the rank interval $\left[r_{1}, r_{2}\right]$ of suffixes that start with $T_{k}[i . . j]$. This can be done in $O(\operatorname{Lookup}(n) \cdot \log n)$ time.

In [18], it was shown how to report for any $1 \leq r_{1} \leq r_{2} \leq n$ all distinct documents $T_{f}$ such that at least one suffix of $T_{f}$ occurs at position $r, r_{1} \leq r \leq r_{2}$, of the generalized suffix array. His construction uses $O\left(n+m \log \frac{n}{m}\right)$ additional bits, and all relevant documents are reported in $O(\operatorname{Lookup}(n) \cdot$ ndocs $)$ time, where ndocs is the number of documents that contain $T_{k}[i . . j]$. Summing up, we obtain the following result.

Theorem 7. All documents containing $T_{k}[i . . j]$ can be reported in $O((\log n+$ ndocs)Lookup $(n))$ time, where ndocs is the number of those. The underlying indexing structure takes $2|C S A|+O\left(n+m \log \frac{n}{m}\right)$ bits of space.

Acknowledgments: T.Starikovskaya has been supported by the mobility grant funded by the French Ministry of Foreign Affairs through the EGIDE agency and by a grant 10-0193109-CNRS-a of the Russian Foundation for Basic Research. Part of this work has been done during a one-month stay of Y.Nekrich at the Marne-la-Vallée University supported by the BEZOUT grant of the French government. 


\section{References}

1. A. Amir, G. M. Landau, M. Lewenstein, and D. Sokol. Dynamic text and static pattern matching. $A C M$ Trans. Algorithms, 3, May 2007.

2. A. Andersson and M. Thorup. Dynamic ordered sets with exponential search trees. J. ACM, 54(3):13, 2007.

3. M. A. Bender, R. Cole, E. D. Demaine, M. Farach-Colton, and J. Zito. Two simplified algorithms for maintaining order in a list. In Proceedings of the 10th Annual European Symposium on Algorithms, ESA '02, pages 152-164, London, UK, UK, 2002. Springer-Verlag.

4. M. A. Bender and M. Farach-Colton. The lca problem revisited. In Proceedings of the 4th Latin American Symposium on Theoretical Informatics, LATIN '00, pages 88-94, London, UK, 2000. Springer-Verlag.

5. M. A. Bender and M. Farach-Colton. The level ancestor problem simplified. Theor. Comput. Sci., $321(1): 5-12,2004$.

6. O. Berkman and U. Vishkin. Finding level-ancestors in trees. J. Comput. Syst. Sci., 48(2):214-230, 1994.

7. P. Bozanis, N. Kitsios, C. Makris, and A. K. Tsakalidis. New upper bounds for generalized intersection searching problems. In Automata, Languages and Programming, 22nd International Colloquium, (ICALP) Proceedings, pages 464-474, 1995.

8. G. S. Brodal, P. Davoodi, and S. S. Rao. Path minima queries in dynamic weighted trees. In Proceedings of the 12th International Symposium on Algorithms and Data Structures, WADS'11, pages 290-301, Berlin, Heidelberg, 2011. Springer-Verlag.

9. P. Dietz and D. Sleator. Two algorithms for maintaining order in a list. In Proceedings of the 19th Annual ACM Symposium on Theory of Computing, STOC '87, pages 365-372, New York, NY, USA, 1987. ACM.

10. M. Farach and S. Muthukrishnan. Perfect hashing for strings: Formalization and algorithms. In Proceedings of the 7th Annual Symposium on Combinatorial Pattern Matching, CPM '96, pages 130-140, London, UK, 1996. Springer-Verlag.

11. M. L. Fredman and D. E. Willard. Trans-dichotomous algorithms for minimum spanning trees and shortest paths. J. Comput. Syst. Sci., 48(3):533-551, 1994.

12. T. Gagie, G. Navarro, and S. J. Puglisi. Colored range queries and document retrieval. In Proceedings of the 17th International Conference on String Processing and Information Retrieval, SPIRE'10, pages 67-81, Berlin, Heidelberg, 2010. Springer-Verlag.

13. A. Golynski, J. I. Munro, and S. S. Rao. Rank/select operations on large alphabets: a tool for text indexing. In Proceedings of the Seventeenth Annual ACM-SIAM Symposium on Discrete Algorithms, SODA 2006, Miami, Florida, USA, January 22-26, 2006, pages 368-373. ACM Press, 2006.

14. R. Grossi and J. S. Vitter. Compressed suffix arrays and suffix trees with applications to text indexing and string matching (extended abstract). In Proceedings of the thirty-second annual ACM symposium on Theory of computing, STOC '00, pages 397-406, New York, NY, USA, 2000. ACM.

15. S. Muthukrishnan. Efficient algorithms for document retrieval problems. In Proc. of the 13th Annual ACM-SIAM Symp. on Discrete Algorithms, January 6-8, 2002, San Francisco, CA, USA, pages 657-666. ACM/SIAM, 2002.

16. G. Navarro and V. Mäkinen. Compressed full-text indexes. ACM Comput. Surv., 39, April 2007.

17. K. Sadakane. Compressed suffix trees with full functionality. Theory Comput. Syst., 41(4):589-607, 2007.

18. K. Sadakane. Succinct data structures for flexible text retrieval systems. J. of Discrete Algorithms, 5:12-22, March 2007. 


\section{Appendix}

Here we prove Theorem 1 . We use the heavy path decomposition technique of [1].

Heavy Path Decomposition. A path $\pi$ in $\mathcal{T}$ is heavy if every node $u$ on $\pi$ has at most twice as many nodes in its subtree as its child $v$ on $\pi$. A tree $\mathcal{T}$ can be decomposed into paths using the following procedure: we find the longest heavy path $\pi_{r}$ that starts at the root of $\mathcal{T}$ and remove all edges of $\pi_{r}$ from $\mathcal{T}$. All remaining vertices of $\mathcal{T}$ belong to a forest; we recursively repeat the same procedure in every tree of that forest.

We can represent the decomposition into heavy paths using a tree $\mathbb{T}$. Each node $\mathbb{v}_{j}$ in $\mathbb{T}$ corresponds to a heavy path $\pi_{j}$ in $\mathcal{T}$. A node $\mathbb{v}_{j}$ is a child of a node $\mathbb{v}_{i}$ in $\mathbb{T}$ if the head of $\pi_{j}$ (i.e., the highest node in $\pi_{j}$ ) is a child of some node $u \in \pi_{i}$. Some node in $\pi_{i}$ has at least twice as many descendants as each node in $\pi_{j}$; hence, $\mathbb{T}$ has height $O(\log n)$.

An $O(n \log n)$ Space Solution. Let $\mathbb{p}_{j}$ denote a root-to-leaf path in $\mathbb{T}$. For a node $\mathbb{v}$ in $\mathbb{T}$ let weight $(\mathbb{v})$ denote the weight of the head of $\pi$, where $\pi$ is the heavy path represented by $\mathbb{v}$ in $\mathbb{T}$. We store a data structure $D\left(\mathbb{p}_{j}\right)$ that contains the values of weight $(\mathbb{v})$ for all nodes $\mathbb{v} \in \mathbb{p}_{j} . D\left(\mathbb{p}_{j}\right)$ contains $O(\log n)$ elements; hence, we can find the highest node $\mathbb{v} \in \mathbb{p}_{j}$ such that weight $(\mathbb{v}) \geq q$ in $O(1)$ time. This can be achieved by storing the weights of all nodes from $\mathbb{p}_{j}$ in the q-heap[11].

For every heavy path $\pi_{j}$ we store the weights of all nodes $u \in \pi_{j}$ in the data structure $E\left(\pi_{j}\right)$; using $E\left(\pi_{j}\right)$, we can find for any integer $q$ the lightest node $u \in \pi_{j}$ such that weight $(u) \geq q$. Using Theorem 1.5 in [2], we can find the above defined node $u \in \pi_{j}$ in $O\left(\sqrt{\log n^{\prime} / \log \log n^{\prime}}\right)$ time where $n^{\prime}=\min \left(n_{h}, n_{l}\right), n_{h}=\mid\left\{v \in p_{j} \mid \operatorname{weight}(v)>\right.$ weight $(u)\} \mid$, and $n_{l}=\mid\left\{v \in p_{j} \mid\right.$ weight $(v)<$ weight $\left.(u)\right\} \mid$. Moreover, we can also find the node $u$ in $O(\log \log q)$ time; we will describe the data structure in the full version of this paper. Thus $E\left(\pi_{j}\right)$ supports queries in $O\left(\min \left(\sqrt{\log n^{\prime} / \log \log n^{\prime}}, \log \log q\right)\right)$ time.

For each node $u \in \mathcal{T}$ we store a pointer to the heavy path $\pi$ that contains $u$ and to the corresponding node $\mathbb{v} \in \mathbb{T}$.

A query wla $(v, q)$ can be answered as follows. Let $\mathbb{v}$ denote the node in $\mathbb{T}$ that corresponds to the heavy path containing $v$. Let $\mathbb{p}_{j}$ be an arbitrary root-to-leaf path in $\mathbb{T}$ that also contains $\mathbb{v}$. Using $D\left(\mathbb{p}_{j}\right)$ we can find the highest node $\mathbb{u} \in \mathbb{p}_{j}$, such that weight $(\mathfrak{u}) \geq q$ in $O(1)$ time. Let $\pi_{t}$ denote the heavy path in $\mathcal{T}$ that corresponds to the parent of $\mathfrak{k}$, and $\pi_{s}$ denote the path that corresponds to $\mathfrak{k}$. If the weighted ancestor wla $(v, q)$ is not the head of $\pi_{s}$, then wla $(v, q)$ belongs to the path $\pi_{t}$. Using $E\left(\pi_{t}\right)$, we can find $u=\operatorname{wla}(v, q)$ in $O\left(\min \left(\sqrt{\log n^{\prime} / \log \log n^{\prime}}, \log \log q\right)\right)$ time where $n^{\prime}=\min \left(n_{h}, n_{l}\right)$, $n_{h}=\mid\left\{v \in p_{t} \mid\right.$ weight $(v)>$ weight $\left.(u)\right\} \mid$, and $n_{l}=\mid\left\{v \in p_{t} \mid\right.$ weight $(v)<$ weight $\left.(u)\right\} \mid$.

All data structures $E\left(\pi_{i}\right)$ use linear space. Since there are $O(n)$ leaves in $\mathbb{T}$ and each path $\mathbb{p}_{i}$ contains $O(\log n)$ nodes, all $D\left(\mathbb{p}_{i}\right)$ use $O(n \log n)$ space.

Lemma 2. There exists a $O(n \log n)$ space data structure that finds the weighted level ancestor $u$ in $O\left(\min \left(\sqrt{\log n^{\prime} / \log \log n^{\prime}}, \log \log q\right)\right)$ time. 
An $O(n)$ Space Solution. We can reduce the space from $O(n \log n)$ to $O(n)$ using a micromacro tree decomposition. Let $\mathcal{T}_{0}$ be a tree induced by the nodes of $\mathcal{T}$ that have at least $\log n / 8$ descendants. The tree $\mathcal{T}_{0}$ has at most $O(n / \log n)$ leaves. We construct the data structure described above for $\mathcal{T}_{0}$; since $\mathcal{T}_{0}$ has $O(n / \log n)$ leaves, $\mathbb{T}_{0}$ also has $O(n / \log n)$ leaves. Therefore all structures $D\left(\mathbb{p}_{j}\right)$ use $O(n)$ words of space. All $E\left(\pi_{i}\right)$ also use $O(n)$ words of space. If we remove all nodes of $\mathcal{T}_{0}$ from $\mathcal{T}$, the remaining forest $\mathcal{F}$ consists of $O(n)$ nodes. Every tree $\mathcal{T}_{i}, i \geq 1$, in $\mathcal{F}$ consists of $O(\log n)$ nodes. Nodes of $\mathcal{T}_{i}$ are stored in a data structure that uses linear space and answers weighted ancestor queries in $O(1)$ time. This data structure will be described later in this section.

Suppose that a weighted ancestor wla $(v, q)$ should be found. If $v \in \mathcal{T}_{0}$, we answer the query using the data structure for $\mathcal{T}_{0}$. If $v$ belongs to some $\mathcal{T}_{i}$ for $i \geq 1$, we check the weight $w_{r}$ of $\operatorname{root}\left(\mathcal{T}_{i}\right)$. If $w_{r} \leq q$, we search for wla $(v, q)$ in $\mathcal{T}_{i}$. Otherwise we identify the parent $v_{1}$ of $\operatorname{root}\left(\mathcal{T}_{i}\right)$ and find wla $\left(v_{1}, q\right)$ in $\mathcal{T}_{0}$. If wla $\left(v_{i}, q\right)$ in $\mathcal{T}_{0}$ is undefined, then wla $(v, q)=\operatorname{root}\left(\mathcal{T}_{i}\right)$.

A Data Structure for a Small Tree. It remains to describe the data structure for a tree $\mathcal{T}_{i}$, $i \geq 1$. Since $\mathcal{T}_{i}$ contains a small number of nodes, we can answer weighted level ancestor queries on $\mathcal{T}_{i}$ using a look-up table $V . V$ contains information about any tree with up to $\log n / 8$ nodes, such that node weights are positive integers bounded by $\log n / 8$. For any such tree $\widetilde{\mathcal{T}}$, for any node $v$ of $\widetilde{\mathcal{T}}$, and for any integer $q \in[1, \log n / 8]$, we store the pointer to wla $(v, q)$ in $\widetilde{\mathcal{T}}$. There are $O\left(2^{\log n / 4}\right)$ different trees $\widetilde{\mathcal{T}}$ (see e.g., [5] for a simple proof); for any $\widetilde{\mathcal{T}}$, we can assign weights to nodes in less than $(\log n / 8)$ ! ways. For any weighted tree $\tilde{\mathcal{T}}$ there are at most $(\log n)^{2} / 64$ different pairs $v, q$. Hence, the table $V$ contains $O\left(2^{\log n / 4}(\log n)^{2}(\log n / 8) !\right)=o(n)$ entries. We need only one look-up table $V$ for all mini-trees $\mathcal{T}_{i}$.

We can now answer a weighted level ancestor query on $\mathcal{T}_{i}$ using reduction to rank space. The rank of a node $u$ in a tree $\mathcal{T}$ is defined as $\operatorname{rank}(u, \mathcal{T})=\mid\{v \in \mathcal{T} \mid \operatorname{weight}(v) \leq$ weight $(u)\} \mid$. The successor of an integer $q$ in a tree $\mathcal{T}$ is the lightest node $u \in \mathcal{T}$ such that weight $(u) \geq q$. The $\operatorname{rank} \operatorname{rank}(q, \mathcal{T})$ of an integer $q$ is defined as the rank of its successor. Let $\operatorname{rank}(\mathcal{T})$ denote the tree $\mathcal{T}$ in which the weight of every node is replaced with its rank. The weight of a node $u \in \mathcal{T}$ is not smaller than $q$ if an only if $\operatorname{rank}(u, \mathcal{T}) \geq \operatorname{rank}(q, \mathcal{T})$. Therefore we can find wla $(v, q)$ in some $\mathcal{T}_{i}$ as follows. For every $\mathcal{T}_{i}$ we store a pointer to $\widetilde{\mathcal{T}}_{i}=\operatorname{rank}\left(\mathcal{T}_{i}\right)$. Given a query wla $(v, q)$, we find $\operatorname{rank}\left(q, \mathcal{T}_{i}\right)$ in $O(1)$ time using a q-heap [11]. Let $v^{\prime}$ be the node in $\widetilde{\mathcal{T}}_{i}$ that corresponds to the node $v$. We find $u^{\prime}=\operatorname{wla}\left(v^{\prime}, \operatorname{rank}\left(q, \mathcal{T}_{i}\right)\right)$ in $\widetilde{\mathcal{T}}_{i}$ using the table $V$. Then the node $u$ in $\mathcal{T}_{i}$ that corresponds to $u^{\prime}$ is the weighted level ancestor of $v$. 